\title{
Los aspectos negativos de vínculos cercanos aumentan el riesgo de enfermedad coronaria
}

\author{
Negative aspects of close relationships are associated with and increased risk of heart disease
}

\section{Objetivo}

Analizar la asociación entre aspectos negativos de vínculos cercanos (ANVC) y eventos coronarios (EC).

\section{Diseño, lugar y pacientes}

Estudio prospectivo de cohortes en 20 departamentos de servicios públicos londinenses que incluyó 9011 pacientes $(2897$ mujeres) pertenecientes a un estudio que había incluído a todos los trabajadores no industriales de 35 a 55 años empleados en dichos departamentos. De los 10.308 encuestados se tomaron para el presente estudio los 9011 que contaban con información disponible acerca de vínculos cercanos. Se excluyeron aquellos que presentaban historia previa de enfermedad cardiaca.

\section{Factores pronósticos}

Se utilizó el "Cuestionario de Personas Cercanas" que incluye 15 items sobre ANVC y soporte emocional e instrumental recibido durante los últimos 12 meses. Si bien se interrogó sobre las cuatro relaciones consideradas más cercanas, solo se tuvo en cuenta para el estudio a la persona definida por el paciente como la más cercana.

\section{Medición de resultado principal}

Incidencia de EC durante un seguimiento promedio de 12,2 años: infarto de miocardio fatal y no fatal o reportes médicos de angina de pecho. Se excluyeron los eventos autoreportados.

\section{Resultados principales}

De los 8499 pacientes libres de EC al comienzo del estudio, 589 los desarrollaron. La vivencia de relaciones interpersonales adversas fue mas frecuentemente reportada por los jóvenes, las mujeres, los hombres con bajo nivel de empleo, y menos reportada por quienes nunca habían estado casados.
De Vogli R y col. Arch Intern Med. 2007;167 (18):1951-1957 Luego de ajustar por edad, sexo, estado civil, nivel de empleo, obesidad, hipertensión, diabetes mellitus, nivel de colesterol, apoyo social y recursos de apoyo, se observó una asociación con efecto dosis-respuesta entre las relaciones cercanas altamente estresantes y la incidencia de EC $\left(\mathrm{HR}^{*} 1,34\right.$; IC95\% 1,10 a 1,63). Luego de ajustar por problemas afectivos y depresión la relación se atenuó pero siguió siendo estadísticamente significativa (HR 1,25; IC95\% 1,02 a 1,55). Ver tabla 1.

Tabla 1: asociación entre los aspectos negativos de los vínculos cercanos (en tercilos de magnitud autoreportada) depresión y el riesgo de eventos coronarios.

\begin{tabular}{c|c|c}
\hline \multirow{2}{*}{$\begin{array}{c}\text { Aspectos negativos de } \\
\text { vínculos cercanos }\end{array}$} & Iercilo & Riesgo relativo e IC95\% \\
\cline { 2 - 3 } & Intermedio & 1 (referencia) \\
\hline & Superior & $1,19(0,97$ a 1,46$)$ \\
\hline Depresión & & $1,34(1,1$ a 1,63$)$ \\
\hline
\end{tabular}

No se evidenció asociación con el sexo y el nivel de empleo. La relación entre ANVC y EC fue mayor en aquellos en quienes la pareja era su vínculo más cercano y en quienes contaban con un mayor nivel de apoyo emocional e instrumental; pero en los individuos viudos, divorciados o separados la misma no alcanzó significación estadística. El nivel de apoyo emocional o instrumental recibido no se asoció con un aumento de la incidencia de EC.

\section{Conclusiones}

Los ANVC aumentan la incidencia de EC.

Palabras clave: enfermedad coronaria, vínculos cercanos, apoyo emocional, estudio prospectivo. Key words: coronary heart disease, close relationships, emotional support, prospective study. Fuente de financiamiento: No reportada.

\section{Comentario}

Numerosos estudios han documentado asociación entre problemas psicosociales y enfermedades biomédicas, por ejemplo, trastornos de ansiedad con enfermedades crónicas, y depresión con EC ${ }^{123}$. Las relaciones interpersonales -pareja, familia y ámbito laboral- también han evidenciado su influencia sobre el desarrollo y el mantenimiento de la depresión, como sobre el cuidado de pacientes de pacientes con patologías que requieren cuidados prolongados ${ }^{4}$. Los contextos familiares y sociales interactúan permanente y recursivamente, sobre los procesos biológicos y emocionales, permitiendo esta visión tener una dimensión más acabada sobre su impacto en la salud. Sin embargo a pesar de este cúmulo de evidencias los procesos interaccionales no figuran dentro de las categorías de diagnóstico psiquiátrico clásico como las del Manual Diagnóstico y Estadístico de los Trastornos Mentales (en inglés: DSMIV). Numerosos estudios -inclusive ensayos clínicos- han demostrado que estrategias de tratamiento como la terapia de pareja pueden ser utilizadas positivamente como tratamiento de la depresión, superando inclusive a la utilización de medicación ${ }^{5}$. Este estudio encuentra una asociación entre aspectos negativos de los vínculos más cer- canos -que fue la pareja en el $70 \%$ de los casos- y el riesgo de EC. Ya existía evidencia de que una pobre relación de pareja era factor de mal pronóstico en mujeres que habían padecido un infarto de miocardio $0^{6}$. Lo mismo sucede con el desarrollo de síndrome metabólico, lo que revela el efecto de los vínculos sobre los hábitos saludables y la adherencia ${ }^{7}$. La relación entre factores emocionales y su relación con $\mathrm{EC}$ hace pensar en un análisis de las causalidades complejas que exigen pensar en términos de interacciones. Por ejempo, una mala relación de pareja se relaciona con depresión, con altos niveles estrés, con estilos particulares de afrontamiento y con baja autoestima; lo que puede asociarse con alteraciones autonómicas, neuroendocrinas y de coagulación ${ }^{8}$.

\section{Conclusión de la comentadora}

El efecto de las relaciones impersonales sobre la EC refuerza la importancia del enfoque biopsicosocial, no solo para explorar la dolencia del paciente sino también para tener en cuenta que la afectación de una esfera puede simultáneamente provocar daño objetivable sobre otra.

María Alejandra Alcuaz [ Grupo de Prácticas de Medicina Familiar. Cooperativa Limitada. alalcuaz@hotmail.com ] Augusto Granel [ Servicio de Medicina Familiar y Comunitaria. Hospital Italiano de Buenos Aires. ] (18):1951-1957.PMID: 17923594. Disponible en URL: http://archinte.ama-assn.org/cgi/reprint/167/18/1951 (último acceso 05/08/08).

\section{Referencia}

1. Michael F y col. Generalized Anxiety Disorder. Am Fam Physician. 2000 Oct 1;62(7):1591-1602

2. Todaro Jy col. Effect of negative emotions on frequency of coronary heart disease (the Normative Aging Study). Am J Cardiol Oct 15, 2003;92:901-6. Citado en sección Tips from other journals de Am Fam Physician. 2004 May 15;69(10).

3. Blumenthal J y col. M. Depression as a risk factor for mortality after coronary artery bypass surgery. The Lancet 2003, 362:604-609.

4. Joiner T y col. The interactional nature of depression. American Psychological Association, edition 2002

5. Leff J y col. The London Depression Intervention Trial: an RCT of antidepressants versus couple therapy in the treatment and maintenance of people with a partner. British Journal of Psychiatry; 2002; 177:95-100

6. Orth-Gomer K y col. Marital stress worsens prognosis in woman with coronary heart disease. JAMA. 2000; 284:3008-3014

7. Troxler W y col. Marital quality and the occurrence of the metabolic syndrome in women. Arch Intern Med. 2005; 156:1022-1027.

8. Ewens B y col. Protective and damaging effects of stress mediators. N Engl J Med. 1998;338:171-179 